Article

\title{
Durable Superomniphobic Surface on Cotton Fabrics via Coating of Silicone Rubber and Fluoropolymers
}

\author{
Arsheen Moiz, Rajiv Padhye and Xin Wang * (ID \\ Centre for Materials Innovation and Future Fashion, School of Fashion and Textiles, RMIT University, \\ Melbourne 3056, Australia; s3494882@student.rmit.edu.au (A.M.); rajiv.padhye@rmit.edu.au (R.P.) \\ * Correspondence: xin.wang@rmit.edu.au
}

Received: 11 February 2018; Accepted: 14 March 2018; Published: 15 March 2018

\begin{abstract}
Performance textiles that protect human from different threats and dangers from environment are in high demand, and the advancement in functionalization technology together with employing advanced materials have made this an area of research focus. In this work, silicone rubber and environmentally friendly fluoropolymers have been employed to explore superomniphobic surface on cotton fabrics without compromising comfort much. It has been found that a cross-linked network between the rubber membrane and the fluoropolymers has been formed. The surface appearance, morphology, handle, thickness and chemical components of the surface of cotton fabrics have been changed. The coated fabrics showed resistance to water, aqueous liquid, oil, chemicals and soil. The comfort of the coated fabrics is different to uncoated cotton fabrics due to the existence of coated layers on the surface of cotton fabrics. This work would benefit the development and design of the next generation of performance textiles with balanced performance and comfort.
\end{abstract}

Keywords: superomniphobic; coating; silicone rubber; cotton

\section{Introduction}

Protective clothing has been developed to help humans withstand hazards and threats from environment (cold, hot, wind, water, soil, gas, etc.) or other sources (fire, explosion, corrosive chemicals, pollution, etc.) [1,2]. With different products developed, protective clothing with a single function, such as being flame retardant [3,4], water repellent/superhydrophobic [5], oil repellent [6], self-cleaning [7], or with cold protection [8], chemical protection [7] and moisture management, has been widely developed to serve customers in different areas [3,9]. Recent research and development are focusing on multiple protection of performance textiles [10], among which the exploration of the omniphobic surface of textiles has been regarded as the key to develop textiles that protect skin against chemicals, oils and lubricants [11].

The development of new fibers and/or the functionalization of current fibers are the pathways to advance technology towards the next generation of performance textiles. High performance fibers are developed with their intrinsic properties being to fulfill the heat and fire protection needs, such as protective clothing, commercial fabrics used in liners, curtain and transport materials, medical and military garments [12], Kevlar for ballistic resistance [13], carbon fiber [14] and nanofibers [15]. However, most protection would need a special fabric surface with special surface and interface function. Much attention has been put on the functionalization of fabric surface for the development of performance textiles, and different technologies have been developed including plasma-enhanced chemical vapor deposition [16], plasma [17], grafting [18] and coating [19].

Coating is regarded as the most feasible and applicable technology for developing performance surface on fabrics. The coated materials and the chemical reaction together with the developed surface structure are the determining factors to the targeted performance. Easy conducting and 
feasibility of industrial application are the aims of developing coating technology, and thus robust coating technology including knife coating [20], dip coating [21], electrospining coating [22] and combined coating [23] have been developed. Due to the intrinsic properties of the coated materials, the coated layer usually exhibits a low surface tension to withstand different substances. Polyurethane, silicone rubber and fluorocompounds are the most widely used materials in functional coating of textiles $[24,25]$. The development of Gore-Tex ${ }^{\circledR}$, in which the polytetrafluoroethylene (PTFE) film is used to functionalize textiles, has seen big success [26]. However, some fluorine-based substances, such as perfluorooctane sulfonate (PFOS) and perfluorooctanoic acid (PFOA) of carbon chains greater than six, are restricted in functional coating of textiles due to the health issues these compounds cause to human. Related policy to ban the using of harmful fluoro-based compounds have implemented and searching for replacement of fluoropolymers is on the way of developing protective clothing [27]. In fact, recent research has contributed to environmental friendly fire repellent solutions for layer-by-layer coating or biomacromolecular assembly on cotton fabrics [28-31].

A superomniphobic surface of the coated layers on fabrics can withstand a series of agents, and this is usually achieved through the combination of materials and the combined action of different coating methods. Previous endeavors have found that the combination of polyurethane with polydimethylsiloxane and trimethylated silica exhibits versatile protection on cotton fabrics [20] and polycotton fabrics [32]. There are many environmentally friendly compounds for the coating of a superomniphobic surface, such as fluoropolymers oleophobol [33] and phobol and silane compound trimethylmethoxysilane (TMMS). The oleophobol ${ }^{\circledR}$ CPR is a C6-chemistry-based perfluorinated polymer compound, and it is superior to the previously used C8-based fluorocarbons that release PFOS and PFOA. In addition, the phobol ${ }^{\circledR} \mathrm{CP}-\mathrm{C}$ is a fluorine-efficient short-chain $\mathrm{C} 6$ chemical repels. This work focused on the combined coating of silicone rubber membrane and environmental friendly fluoropolymers to develop the superomniphobic surface on cotton fabrics. The coated fabrics were characterized by chemical analysis, handle, morphology and protection against different agents including water, oil, aqueous liquid, chemicals and soil. Besides, the comfort of the coated fabrics was studied to better understand the effect of the coating on clothing development. The developed coating technology would benefit the development of the next generation performance textiles for versatile protection.

\section{Experimental}

\subsection{Materials}

Cotton fabrics $(30 \mathrm{~cm} \times 30 \mathrm{~cm})$ were purchased from Bruck Textiles, Australia. Isys HPx was purchase from CHT Pty Ltd., Dandenong, Australia. Rubber membrane (SILASTIC 1951-200P, LSR), in paste form, was purchased from Dow Corning Europe S. A, Belgium. Invadine PBN, Kinttex ${ }^{\circledR}$ FEL, Phobotex RSH, Phobol ${ }^{\circledR} \mathrm{CP}-\mathrm{C}$ and Oleophobol ${ }^{\circledR} \mathrm{CP}-\mathrm{C}$ were purchased from Huntsman Singapore Pty Ltd., Gateway West, Singapore. Trimethylmethoxysilane (TMMS), Hexadecane and N-Decane were purchased from Sigma Aldrich Pty Ltd., Australia. Mineral oil was purchased from Johnson \& Johnson Pacific Pty Ltd., Sydney, Australia. Acetonitrile, isopropyl alcohol and dichloromethane (Ana-R) were purchased from BDH Limited Poole, England. Sulphuric acids (98\%) was purchased from RSL LAB SCAN Ltd., Bangkok, Thailand. Dimethylformamide was purchased for Merck, Germany. Sodium hydroxide was purchased from Chem Supply Pty Ltd., Gillman, Australia.

\subsection{Methods}

A conventional dipping-padding-knife-curing method [20,32] was applied to perform the coating on cotton fabrics $(30 \mathrm{~cm} \times 30 \mathrm{~cm})$, as shown in Figure 1a. Fabrics were scoured in a MCS mini-jet dyeing vessel for $20 \mathrm{~min}$ at $60^{\circ} \mathrm{C}$. The solution in the vessel had a liquor ratio of 20:1 with $w / v$ Triton X100. Scoured fabrics were rinsed in warm water and then in cold water. Rinsed fabrics were left overnight for hanging dry in an ambient environment. To impart hydrophobic properties on fabrics, 
various chemical coatings were applied on the fabric using an Ernst Benz Pad machine. Fabrics were passed through the machine at a pad speed of $2 \mathrm{~m} / \mathrm{min}$ at $8 \mathrm{kPa} / \mathrm{cm}$ pressure. While padding, $10 \%$ solution of the chemical was applied and each fabric sample was passed through the pad three times to ensure even layering of the chemical on the fabric surface. These samples were then dried at $60{ }^{\circ} \mathrm{C}$ for $30 \mathrm{~min}$ and cured at $160^{\circ} \mathrm{C}$ for $5 \mathrm{~min}$.

At first, cotton fabrics (A) were padded with a single layer of the chemical solutions on fabric surface. As shown in Figure 1b, solution of $5 \mathrm{~g} / 100 \mathrm{~mL}$ of Isy Hpx with $95 \mathrm{~mL}$ distilled water was applied on the fabric surface to test its reaction. The solution acted as a cation-crosslinking agent to network the repellent chemical layers on the fabric. A single layer of rubber membrane was coated on the padded fabrics by knife-edge coating method. The coating consisted of LSR (part a [90 g] + part b $[10 \mathrm{~g}]$ ) and was applied uniformly on the fabrics (B). Using the same padding method, fluoropolymers Phobol CP-C (10\% Solution) and Oleophobol CP-C (10\% solution) were applied on the fabric surface and a percentage pickup of the chemical after padding on the cotton fabric was calculated. The further padded fabrics were denoted as sample $C$ and $D$, respectively. Alternatively, a double layer of TMMS + Phobol was padded onto the Isys Hpx padded fabric to make sample E.

In order to study the combination of rubber membrane and fluoropolymers, three different samples were prepared by applying multi-layer coating on surface of cotton fabrics (Figure 1b). It included triple layers of Isy Hpx + rubber membrane + Phobol (F), Isy Hpx + rubber membrane + Oleophobol (G) and Isy Hpx + Rubber membrane + TMMS + Phobol (H).

(a)

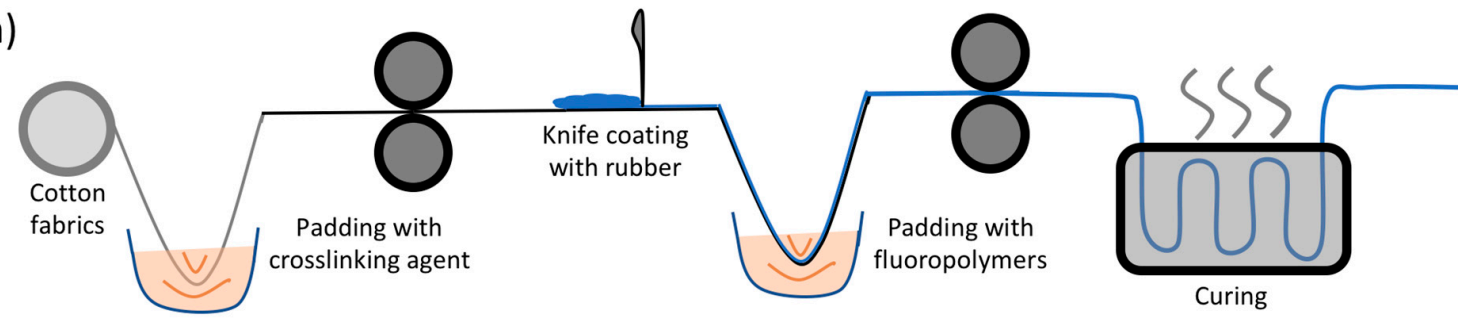

(b)

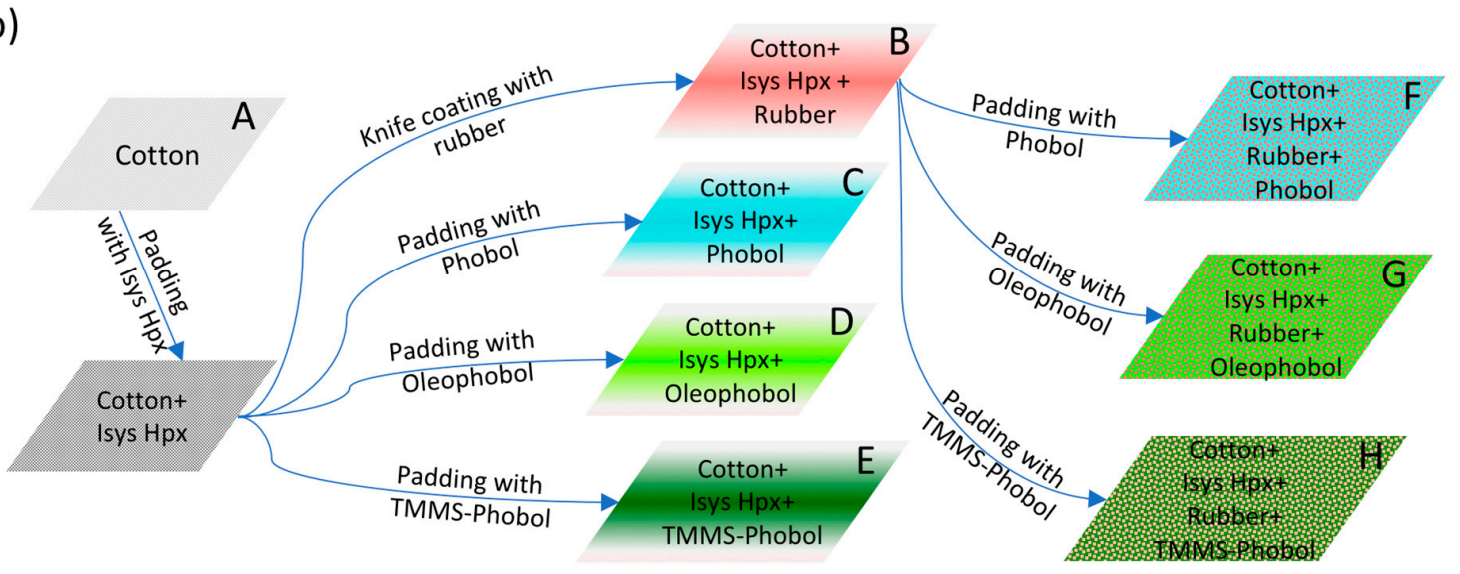

Figure 1. Schematics of the pad-knife-pad-cure method (a) and the experimental design (b).

\subsection{Characterization and Measurements}

\subsubsection{Scanning Electron Microscopy}

A field emission scanning electron microscope (FESEM, Quanta ${ }^{\mathrm{TM}}-200$, Oxford, UK) was used to obtain the surface morphology of the coated and uncoated fabrics. The instrument was set at a pressure of 0.65 Torrs and a voltage of $20 \mathrm{keV}$ at room temperature. The samples were prepared by sputter coating (IMBROSE, Spi A20015, Spi Supplies, West Chester, USA) a thin layer of gold particles. 


\subsubsection{Thickness}

The thickness of the coated fabrics was calculated on a thickness meter according to the standard AS 4878.4-2001.

\subsubsection{FTIR Spectroscopy}

An attenuated total reflection Fourier Transform infrared spectroscopy (ATR-FTIR) spectrum was reported for each of the samples using a spectrophotometer (Perkin Elmer 400, Beaconsfield, UK). In the test, a piece of fabric was placed on the ATR crystal and the scanning was running with the range from $4000-650 \mathrm{~cm}^{-1}$.

\subsubsection{Handle}

The handle of the fabrics was conducted by a subjective evaluation of the physical properties of the fabrics by the sense of touch. The flexibility, thickness, luster and smoothness can be compared between different fabrics. The stiffness, surface friction and roughness were measured objectively on different testers according to specific testing standards. In specific, the stiffness rest was performed according to the standard ASTM D1388-14. Four specimens with the size of $25 \mathrm{~mm} \times 75 \mathrm{~mm}$ were placed along the horizontal direction on the flat surface of the stiffness tester with its edge matched with right hand side of the tester marked as zero. The slide was then slightly moved for the specimen to touch the knife edge at an angle of $41.5^{\circ}$. The overhanging length of fabric was recorded as the bending length, and flexural rigidity was calculated from the bending length and the fabric weight accordingly. The surface friction and roughness were measured by the Kawabata evaluation system (KES). An automatic surface tester (Katotech Corp., Kyoto, Japan) was used to measure the fabric softness, shearing, bending, compression and surface friction and roughness. During the test, the parameter of tension was set as $20 \mathrm{gf} / \mathrm{cm}$ and the weight load was approximately $400 \mathrm{~g}$. Three specimens with the size of $20 \times 20 \mathrm{~cm}$ were tested from both warp and weft directions. The maximum sweep of fabric was $2.5 \mathrm{~cm}$ at a speed of $1 \mathrm{~mm} / \mathrm{s}$ on the pile. The coefficient of surface friction (MIU), mean deviation of MIU (MMD) and geometrical roughness (SMD) were reported in $\mu \mathrm{m}$. Each sample was measured by three times and the results were averaged.

\subsubsection{Water Contact Angle}

The water contact angle was measured on a contact angle system (Data physics, OCA20, Starnberg, Germany) at room temperature. A fixed needle was mounted $10 \mathrm{~mm}$ above the fabric sample and a droplet of $5 \mu \mathrm{L}$ of ultra-pure water was placed on the fabric through the Milli-Q filtration system. Picture was taken to calculate the contact angle by the sessile drop method with each sample measured five times.

\subsubsection{Oil Repellency}

According to the standard AATCC 118:2013, three specimens $(2 \mathrm{~mm} \times 2 \mathrm{~mm})$ were dipped into the beaker one by one and a $10.0 \mathrm{~mL}$ oil solution was placed on the fabrics to do the test. Observations were done at 45 degrees to the fabrics and photos were taken for all kinds of oils after $10 \mathrm{~s}$ and $600 \mathrm{~s}$, respectively. Six types of oils were used, namely the paraffin oil in green color, castor oil in colorless, vegetable oil in yellow, $n$-hexadecane in blue color, $n$-decane in red color and $n$-heptane in orange color.

\subsubsection{Aqueous Liquid Repellency}

According to the standard AATCC 193:2012, a 10- $\mu$ L droplet of isopropyl alcohol was dropped onto the three specimens $(5 \mathrm{~cm} \times 5 \mathrm{~cm})$ in a Petri dish. Photos of the droplets were taken from a 45-degree angle to observe the repellency of the fabrics. The grade was recorded if the droplet was repelled after $10 \mathrm{~s}$ and $600 \mathrm{~s}$, otherwise a zero was recorded if the droplet penetrated into the fabrics. 
Different percentages of water/alcohol mixture were used, namely the 98/2 W/A in orange color, $90 / 10 \mathrm{~W} / \mathrm{A}$ in red color, $80 / 20 \mathrm{~W} / \mathrm{A}$ in purple color and 60/40 W/A in yellow color.

\subsubsection{Chemical Resistance}

The chemical resistance test was performed to measure the chemical resistance of the uncoated and coated fabrics. Three specimens $(2 \mathrm{~mm} \times 2 \mathrm{~mm})$ were placed in a Petri dish and three $10.0 \mu \mathrm{L}$ droplets of the chemicals were placed on the fabric specimens. The resistance of chemicals was observed at $45^{\circ}$ after 10 and $600 \mathrm{~s}$, respectively. Sixteen types of chemicals were used to repeat the test. The time interval of each chemical was recorded in seconds otherwise zero means the chemical penetrated into the fabrics.

\subsubsection{Soil Resistance}

The soil resistance was measured with the stain on the fabrics, according to the standard method AATCC-130-2015 with minor change of replacing oil (corn oil) with water. Soil particles (5.0 g) were dissolved into $5.0 \mathrm{~mL}$ of water to make the solution. Three specimens with the size of $50 \mathrm{~cm} \times 100 \mathrm{~cm}$ were used to determine the soil staining. The specimen was mounted on a glass slide at $45^{\circ}$ with the help of paper clips. Soil resistance was measured into five grades of equivalent staining for $20 \mathrm{~s}$ by comparing the observed stain to the standard stains.

\subsubsection{Laundering Test}

According to the standard AATCC 61:2013(1A), the sample fabrics were washed by the AATCC reference detergent in a SDL Atlas Launder-O-meter at $40{ }^{\circ} \mathrm{C}$ with the presence of 10 steel balls. Each specimen was rinsed twice in deionized water followed by a drying process in an air circulating oven for $30 \mathrm{~min}$. After 30 cycles of laundering, the water contact angle of the fabrics was tested to investigate the durability of the coatings.

\subsubsection{Water Repellency}

According to the standard AATCC 22:2010, a sample with the size of $180 \mathrm{~mm} \times 180 \mathrm{~mm}$ was mounted between the test hoops to undergo spraying of $250 \mathrm{~mL}$ of distilled water for over $30 \mathrm{~s}$ and the spray rate was obtained.

\subsubsection{Air Permeability}

Air permeability was measured in accordance with the standard AS-2001.2.34:1990 on an air permeability tester (SDL Atlas Pty Ltd., Stockport, UK). The test was performed ten times at a pressure of $100 \mathrm{kPa}$, and a tube no- $04(\mathrm{~L} / \mathrm{min})$ with small orifice diameter of $2.8 \mathrm{~cm}^{2}$ was used.

\subsubsection{Pilling Resistance}

Pilling was measured by a Martindale Pilling Tester (IDM Instrument Pty Ltd., Hallam, Australia), in accordance with the standard ISO 12945-2:1998. The specimen and a foam were fixed in the given plates and the specimen was subject to rubbing for 50, 500, 1000 and 2000 cycles. After the completion of each pilling cycles, the water contact angle was measured to assess the durability of the coatings.

\subsubsection{Thermal Resistance}

A Sweating Guarded Hotplate (SDL Atlas Pty Ltd., Stockport, UK) was used to measure the thermal resistance $\left(R_{c t}\right)$ in accordance with the standard ISO11092:1993(E). Three specimens $(30 \mathrm{~cm} \times 30 \mathrm{~cm})$ were placed on the measuring plate with the measuring unit temperature $\left(\mathrm{T}_{\mathrm{m}}\right) 35^{\circ} \mathrm{C}$, air temperature $\left(\mathrm{T}_{\mathrm{a}}\right) 20{ }^{\circ} \mathrm{C}$, air circulation speed $1 \mathrm{~m} / \mathrm{s}$ and $\mathrm{RH} 65 \%$. The thermal resistance was reported automatically on the screen after the completion of the test. 


\subsubsection{Water Vapor Resistance}

On the same sweating guarded hotplate (SDL Atlas Pty Ltd., Stockport, UK), the water vapor resistance $\left(R_{e t}\right)$ was measured according to the standard ISO11092:1993(E). The speed of the circulation air was $1 \mathrm{~m} / \mathrm{s}$ in the chamber and the power consumed to maintain the temperature of the plate at $35^{\circ} \mathrm{C}$ for $15 \mathrm{~min}$ was measure. The permeability index $\left(\mathrm{I}_{\mathrm{m}}\right)$ was calculated from the value of $\mathrm{R}_{\mathrm{ct}} / \mathrm{R}_{\mathrm{et}}$.

\subsubsection{Moisture Management Property}

A SDL Atlas Moisture Management Tester (MMT) was used to measure the moisture management property of fabrics according to the standard AATCC-TM-195 (2009). The tested fabric $(80 \mathrm{~cm} \times 80 \mathrm{~cm})$ was put on the test platform and a saline solution was penetrating from the top to the bottom of the fabric. The MMT profile of the fabric was then reported on an associated computer.

\section{Results and Discussion}

A three-layer coating was added on the surface of cotton fabrics followed by a curing process to initiate the crosslinking between the layers, as illustrated in Figure 2a the mechanism of the coating. The first padding of Isy Hpx added the crosslinking agent onto the cotton fabrics. The second and third steps of the coating added silicone rubber membrane and fluoropolymers onto the padded cotton fabrics, respectively. The following curing procedure was essential to initiate the crosslinking process, resulting in a crosslinked network with durability. Strong bonding between the $\mathrm{CH}_{3}$ groups of rubber and the $\mathrm{CF}_{2}$ groups of fluoropolymers has been established after the curing process, bringing durability of the coated layers. As a result, the surface appearance, morphology, handle, thickness and chemical components of the surface of cotton fabrics have been changed. Meanwhile, the protection of the surface has been enhanced with comfort compromised to a certain level.

\subsection{Characterization}

\subsubsection{Morphology}

Figure $2 \mathrm{~b}$ shows the optical photos of all the samples. It is evident that rubber coating has resulted in a membrane with shiny appearance on the surface of fabrics, as shown the optical photos of samples B, F, G and H. Whereas, the padding of fluoropolymers (samples C, D and E) have not affected the fibrous structure much as compared to uncoated cotton fabrics (sample A).

Scanning electron Microscope (SEM) photos have further confirmed the surface morphology of all the samples, as shown in Figure $2 b$. The typical morphology of cotton fibers is shown in the photo for sample A, indicating the convolutional ribbon profile of cotton. The samples padded with fluoropolymers (samples C, D and E) show clear fibrous structures, but the structure of these samples is more compact than that of sample A. The squeezing involved in the padding process has forced the fibers together with fluoropolymers impregnated into the fibrous structures, resulting in the compacted structure of the padded samples. On the other hand, samples B, F, G and H show a membrane on their surface with some fibers and particles, and the fibrous structure has been severely altered by coating of rubber. A quite uniform membrane has been formed on the surface of fabrics after coating with rubber, and it is evident that this layer will act as the barrier to withstand water, oil and chemical from penetrating onto the fabrics. Unfortunately, the membrane blocks the pores of the fibrous structure, and thus the comfort of the as-coated fabrics will be deteriorated accordingly.

\subsubsection{Thickness}

The thickness of coated fabrics is different from the uncoated fabrics depending on the coating methods and the as-coated materials. Figure $2 \mathrm{c}$ illustrates the thickness of cotton fabrics before and after different scenarios of coating (Figure 1b). The thickness of fabrics after padding usually decreases due to the squishing of the rollers, resulting in thinner fabrics with a more compact fibrous structure. 
As shown the thickness of samples C, D and E in Figure 2a, fabrics after padding ended up with a lower thickness no matter what fluoropolymers were used.
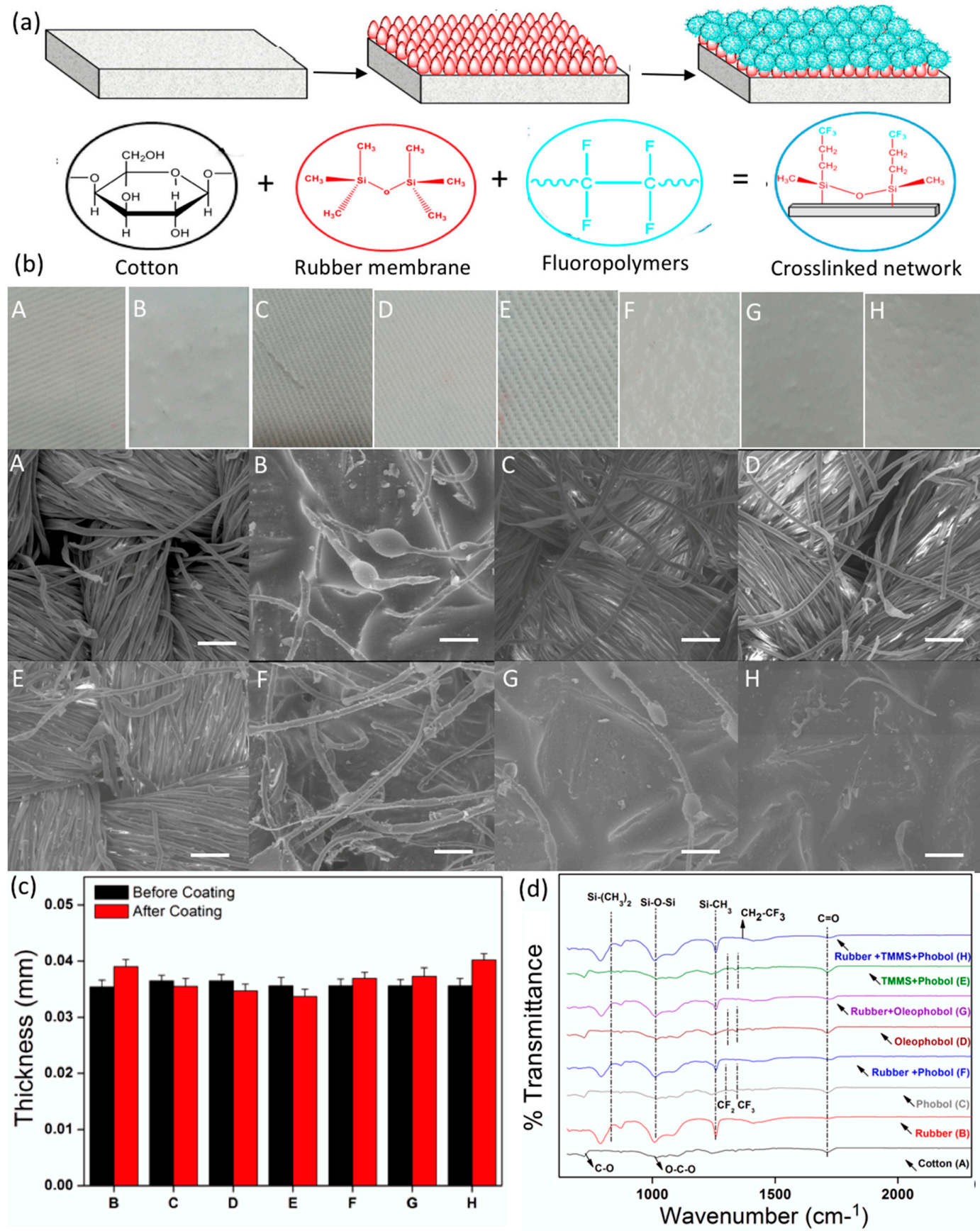

Figure 2. (a) Schematics of the mechanism; (b) optical photos and SEM photos (scale bar $=100 \mu \mathrm{m}$ ); (c) thickness; (d) FTIR spectra.

Knife coating usually results in a uniform membrane on fabric surface with a larger thickness. Cotton fabrics coated by rubber (sample B) show an evidence increase in thickness as indicated in Figure 2a. Further padding of fluoropolymers on the coated rubber surface would reduce the thickness a little bit due to the squeezing effect, so that the thickness of sample $F$ and $G$ is relatively lower than that of sample B. However, sample H shows the highest thickness due to the multilayer of fluoropolymers that were added onto the rubber surface. 


\subsubsection{Handle}

The cotton fabrics exhibit different handle after the padding and coating processes. The stiffness of fabrics is different before and after coating. Cotton fabrics are flexible with a bending length of $2.2 \mathrm{~cm}$ and flexural rigidity $0.12 \mathrm{mg} / \mathrm{cm}$, and this is due to the loose woven structure as shown in Figure $2 \mathrm{~b}$. Padding has ended up with a compact fabric structure for cotton fabrics as suggested by the SEM photos, thus the padded fabrics become inflexible, hard and more stiff [34,35]. As a result, the bending length and flexural rigidity for fluoropolymers padded cotton fabrics (samples C, D and E) are around $2.3 \mathrm{~cm}$ and $0.17 \mathrm{mg} / \mathrm{cm}$, respectively. It is noted that the padding of fluoropolymers has not affected the stiffness much, considering the slightly changed stiffness. However, the coating of rubber has dramatic effect on the stiffness of cotton fabrics. The formation of rubber membrane within and on the surface of the fibrous structure has made the fabrics rigid and stiff. The bending length and flexural rigidity for rubber coated samples (B, F, G, and $\mathrm{H}$ ) have been enhanced to $3.4-3.9 \mathrm{~cm}$ and $1.35-2.22 \mathrm{mg} / \mathrm{cm}$. The coating of rubber plus Phobol (sample F) showed the highest stiffness.

The surface friction and roughness of cotton fabrics are also different after the coatings. The coefficient of surface friction (MIU) and geometrical roughness (SMD) of cotton fabrics (A) and fluoropolymer coated fabrics such as $\mathrm{C}, \mathrm{D}$ and $\mathrm{E}$ are almost the same with the value change around $0.16-0.18 \mu \mathrm{m}$ and $4.0-4.2 \mu \mathrm{m}$ for MIU and SMD, respectively. It is evident that fluoropolymers coating has no obvious effect on the surface friction and roughness of cotton fabrics even though particles of fluoropolymers have been formed on the surface of fabrics. However, with rubber membrane coated (samples B, F, G and H), the surface friction together with geometrical roughness have been increased to $0.2-0.3 \mu \mathrm{m}$ and $5.6-6.4 \mu \mathrm{m}$, respectively [36].

\subsubsection{FTIR Spectra}

The Fourier Transform Infrared (FTIR) spectra of all the samples are depicted in Figure 2d. The cotton fabrics (sample A) represent peaks around $2962 \mathrm{~cm}^{-1}$, attributing to the asymmertic and symmertic stretching of the $\mathrm{C}-\mathrm{H}$ bond of the methyl group. The bands at $3400 \mathrm{~cm}^{-1}$ are assigned to the $\mathrm{O}-\mathrm{H}$ stretching for vibration bonding. The rest bands at 1425, 1250 and $1072 \mathrm{~cm}^{-1}$ are due to the $\mathrm{C}-\mathrm{H}$ bending, $\mathrm{C}-\mathrm{H}$ and $\mathrm{C}-\mathrm{O}-\mathrm{C}$ stretching, respectively. Besides, the bands at $1740 \mathrm{~cm}^{-1}$ are attributed to the $\mathrm{C}=\mathrm{O}$ groups of cotton fibers.

The cotton fabrics coated with rubber membrane (sample B) show the peaks at 1258, 1080, 1009 and $792 \mathrm{~cm}^{-1}$, crosspounding to the $\mathrm{Si}-\mathrm{CH}_{3}$ and $\mathrm{Si}-\mathrm{O}-\mathrm{Si}$ groups of rubber. Individual spectra of phobol, olephobol and TMMS as seen in the Figure 2 (sample $\mathrm{C}$, D and E) present clear peaks of $\mathrm{CF}$ and $\mathrm{CF}_{3}$ groups at around 1338 and $1208 \mathrm{~cm}^{-1}$, respectively. After the coating of rubber membrane combined with the fluoropolymers (sample $\mathrm{F}, \mathrm{G}$ and $\mathrm{H}$ ), the peaks for the functional groups of fluoropolymers $\left(\mathrm{CF}_{2}\right.$ and $\left.\mathrm{CF}_{3}\right)$ have slightly shifted due to the formation of tri-fluoroacetic anhydride $\left(-\mathrm{Si}-\mathrm{CH}_{2}-\mathrm{CF}_{3}\right)$ as a result of their polymerzation reactions with cyclic organosilanes. Fluoropolymers have been involved to make the hydrophobic surface on cotton fabrics as shown in these FTIR spectra $[16,20,21]$. The $\mathrm{CF}_{2}$ and $\mathrm{CF}_{3}$ were deformed and mixed with other rocking bands at 1338 to $1330 \mathrm{~cm}^{-1}$. Other peaks in the range of 1100 to $650 \mathrm{~cm}^{-1}$ represent the bands of fluoropolymers combined with rubber. Overall, surface functionalization of fluorpolymers combined with rubber membrane has been evidenced by the FTIR spectra.

\subsection{Protection}

Due to the combined effect of fluoropolymers and rubber membrane, a superomniphobic surface has been created on the surface of the coated cotton fabrics. The developed performance fabrics showed excellent protection against water, liquids, oils, different chemicals and soil. 


\subsubsection{Water Contact Angle}

Figure 3a illustrates the water contact angle (WCA) of all the coated cotton fabrics with the profiles of the water droplet on each fabric. Cotton fabrics are hydrophilic and a droplet of water can penetrate and sink into the fabrics within a second. The WCA of uncoated cotton fabrics is around $30-50^{\circ}$. The developed coating has apparently made the fabrics resistant to water, and the water droplets can stay on the surface of the as-coated fabrics (as shown the profiles of water droplets in Figure 3a). Coating of rubber has brought a membrane with particles onto the surface of cotton fabrics, the surface roughness has been enhanced and the surface energy been reduced. The WCA for rubber-coated fabrics is $141^{\circ}$ as shown the sample B in Figure 3a. Fluoropolymers have very evident hydrophobic effect and the treated fabrics usually exhibit a water contact angle of around $150^{\circ}$. Phobol, oleophobol and TMMS-phobol were used to generate the surface roughness on cotton fibers, and the treated cotton fabrics show a water contact angle of $145^{\circ}(\mathrm{C}), 147^{\circ}$ (D) and $142^{\circ}(\mathrm{E})$, respectively. The combination of rubber membrane and fluoropolymers (phobol, oleophobol and TMMS-phobol) has resulted in a WCA of $142^{\circ}(\mathrm{F}), 144^{\circ}(\mathrm{G})$ and $145^{\circ}(\mathrm{H})$ for the coated cotton fabrics. The enhancement of WCA to cotton fabrics is due to the low surface tension and the roughness of the coated rubber and fluoropolymers.

Durability of coating is the determine factor of the as-developed performance textiles, because the products are subject to different cycles of washing and different mechanical movements. Figure $3 a$ shows the WCA of all the coated samples after different cycles of laundering. The coating of rubber shows excellent durability as the reduction of WCA is $7.0 \%$ even after 30 cycles of laundering. The durability of rubber coating is probably due to the excellent membrane forming properties of rubber which can withstand repeated washing. Fluoropolymers coatings show poor durability (samples C-E in Figure 3a), and the reduction of WCA is $11.7 \%, 15.0 \%$ and $12.0 \%$, respectively. Fluoropolymers coating results in particles on the surface of cotton fabrics that bring hydrophobicity to the coated fabrics. However, the bonding between cotton fibers and the fluoropolymers is very poor. The poor durability of the fluoropolymers is due to the removal of the formed particles on the surface of cotton fabrics after washing. The combination of rubber and fluoropolymers (samples F-H) show enhanced durability, and the reduction of WCA is $7.0 \%, 9.7 \%$ and $10.3 \%$, respectively. Compared with the reduction of WCA of fluoropolymers coating, the combination of rubber and fluoropolymers has enhanced the reduction of WCA by $4.7 \%, 5.3 \%$ and 1.7\% for phobol, oleophobol and TMMS-phobol, respectively. As discussed in the mechanism, a cross-linked network has been formed between rubber and fluoropolymers after the establishing of the bonding between the $\mathrm{CH}_{3}$ groups of rubber and the $\mathrm{CF}_{2}$ groups of fluoropolymers. The cross-linked network is rather strong to withstand washing; thus, the combination of rubber and fluoropolymers shows enhanced durability.

Figure $3 b$ shows the durability of the coated cotton fabrics after different cycles of pilling. All the samples show excellent durability against pilling, suggesting the strong adhesion between the coated layers and the cotton fabrics to withstand mechanical movements. The rubber membrane shows the best durability against pilling, as the WCA doesn't change much even after 2000 cycles of pilling. Evident reduction of WCA can be seen for the coatings with fluoropolymers while the combination of rubber and fluoropolymers shows enhanced durability as expected. The cross-linked network between rubber and fluoropolymers has better durability when the coated fabrics are subject to pilling, and this is due to strong bonding within the network.

\subsubsection{Water Repellency and Air Permeability}

The hydrophobicity of cotton fiber and the porous fibrous structure make cotton fabrics a low water repellency of 50\% [20]. The water repellency of the coated fabrics is much higher than that of cotton fabrics (Figure 3c), due to the surface hydrophobicity and the altered surface structure. Coating of rubber has resulted in a membrane on the surface of cotton fabrics, and thus the water repellency is as high as $80 \%$. The rubber membrane is not absolutely waterproof, suggesting some pores exist within the membrane to allow the penetration of the $20 \%$ of the water. The water repellency of coated surface with fluoropolymers is $90 \%$ for sample C and D, and $80 \%$ for sample E. The lower 
surface tension of fluoropolymers shows excellent resistance to water as compare to the rubber membrane. Besides, the forming of particles on the surface of fabrics blocks some the pores of the fabrics, so that the water repellency has been enhanced. The combination of rubber and fluoropolymers has not contributed to the enhancement of water repellency further, and the repellency is $80 \%$ and even $70 \%$ for sample $\mathrm{H}$. As the fluoropolymers have been coated on the rubber membrane rather than on the cotton fabrics, the pores from fibrous structures are just covered by the rubber membrane. The blocking effect from the formed particles is not as evident as sample C, D and E, resulting in a relatively lower water repellency.
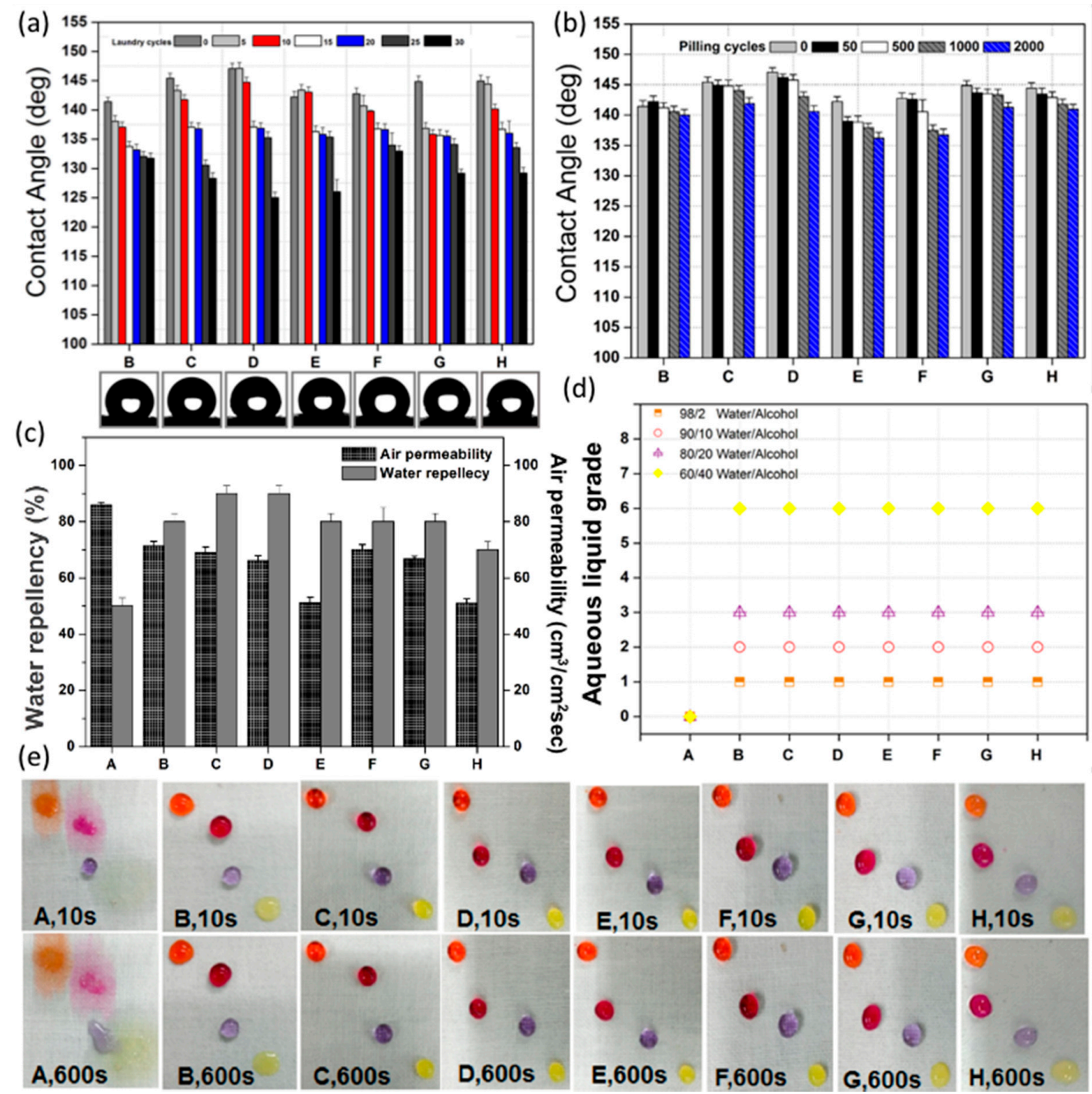

Figure 3. Protection of the as-coated cotton fabrics against water and aqueous liquids: (a) WCA after laundering (Photos: WCA profiles); (b) WCA after pilling; (c) Water repellency and air permeability; (d) Aqueous liquid repellency; (e) Photos of droplets of aquous liquids on the surface of cotton fabrics (Water/alcohol ratios: Organge 98/2; Pink 90/10; Purple 80/20; Yellow 60/40).

The air permeability of cotton fabrics has been affected after coating, as the results show in Figure $3 \mathrm{~d}$. The pore size and the fibrous structure usually determine the air permeability or the breathability of the fabrics $[15,37]$. Cotton fabrics show the air permeability of $86 \mathrm{~cm} / \mathrm{s}$. The coating of rubber membrane on cotton fabrics has resulted in the air permeability of $70 \mathrm{~cm} / \mathrm{s}$. The decline of air permeability is due to the blocking of pores by the rubber membrane. Coating of fluoropolymers has created many particles to block the pores of cotton fabrics, thus the air permeability is similar 
to that of rubber coated one. However, sample E shows the air permeability of $50 \mathrm{~cm} / \mathrm{s}$ as the TMMS-phobol has the maximum blocking effect to the pores within fibrous structure. On the other hand, the air permeability of the combination of rubber and fluoropolymers is similar to that of rubber or fluoropolymer coated ones. Since the fluoropolymers are coated on the surface of rubber membrane, the blocking effect from the formed particles are not evident as that on the surface of cotton fabrics.

\subsubsection{Aqueous Liquid Repellency}

Figure $3 \mathrm{~d}$ shows the aqueous liquid repellency of cotton fabrics before and after coatings, and Figure $3 \mathrm{e}$ lists the photos of the aqueous liquids on the surface of cotton fabrics. Cotton fabrics are not resistant to any aqueous liquids and all the liquids penetrate into the firbous structure within several seconds. Due to the excellent hydrophobicity, the coated samples repel all the aquous liquids for $600 \mathrm{~s}$. The lower surface tension of coated surface usually plays an improtant role in the resistance to aqueous liquid and chemicals [38]. The surface tension of aqueous liquids decreases from 59.0 to 25.4 dynes/cm with the increase of the alcohol/water ratio. As the surface tension of all the coated fabrics are much lower than this, thus all the aqueous liquids are expelled and a near-sphere droplets are shown in the photos of Figure 3e.

\subsubsection{Oil Repellency}

The oil repellency depends upon the surface tension of the coated surface. Uncoated cotton fabric have a high surface tension to enhance the absorbancy of liquids and oil, so that all the oils have penetrated into the fibrous structure as shown in Figure 4a. The rubber membrane and fluoropolymer have low surface tensions to reduce the surface tension of the coated cotton fabrics and thus enhance the oil repellency [20]. As shown in Figure 4a, all the coated cotton fabrics show excellent oil repellency and the oil droplets stay on the surface of fabrics for as long as $600 \mathrm{~s}$. The oil with high surface tensions, such as castor oil 41.35 dynes $/ \mathrm{cm}$, paraffin oil 28.0 dynes $/ \mathrm{cm}, n$-hexadecane 27.5 dynes $/ \mathrm{cm}, n$-decane 23.5 dynes/ $\mathrm{cm}$ and vegtable oil 26.8 dynes/ $\mathrm{cm}$ are repelled by fluoropolymers and their combination with rubber-coated samples C-H. It is evident that the low surface tension of fluoropolymers $(16.2-18.2$ dynes $/ \mathrm{cm}$ ) brings the excellent oil repellency to the coated fabrics. Due to the closer surface tensions between $n$-heptane $(19.8$ dynes $/ \mathrm{cm})$ and the coated fabrics $(18.2$ dynes $/ \mathrm{cm})$, the $n$-heptane is not repelled by most coated fabrics except for sample $\mathrm{E}$ and $\mathrm{H}(16.2$ dynes $/ \mathrm{cm})[6,39]$. However, the difference in surface tension between TMMS (41.0 dynes $/ \mathrm{cm})$ [40] and $n$-heptane is high enough to grant the coated fabrics with repellency to $n$-heptane. On the other hand, rubber coated fabrics are not resistant to $n$-heptane and $n$-decane as shown in Figure $4 \mathrm{a}$, and this is also due to their similar surface tensions. It is evident that fluoropolymers coating has a very evident effect on oil repellency $[34,41]$.

\subsubsection{Chemical Resistance}

Table 1 illustrates the chemical resistance of cotton fabrics to different chemicals, and Figure $4 \mathrm{~b}$ shows the photos the droplets of some chemicals on the all the samples. Cotton fabrics are not resistant to chemicals due to the wettability of cotton, and all the chemicals have been absorbed into the fibrous structure immediately with wet marks left on the surface of fabrics.

The coated cotton fabrics are resistant to most chemicals except for dichloromethane, $n$-hexane and tetrahydrofuran. Due to the extremely low surface tension, fluoropolymers are more effective in resisting chemicals than rubber. Fluoropolymers-coated cotton fabrics show chemical resistance to $n$-decane, sulphuric acids, $n$-heptane, trimethylamine and toluene, while that of rubber-coated membranes is not resistant to these chemicals. Besides, the combination of rubber and fluoropolymers is slightly more effective than fluoropolymers. The combination of rubber membrane and fluoropolymers has generated an omniphobic surface to display the optimized chemical resistance as compare to the uncoated surface of cotton fabrics $[7,11,20,32]$. 
(a)

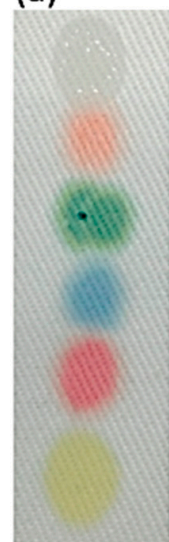

A, 600

(b)

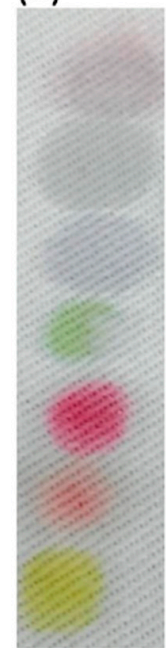

A, 600

(c)

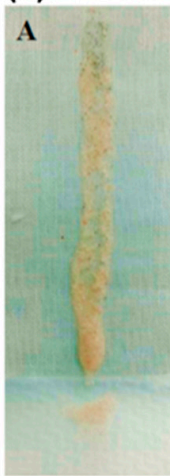

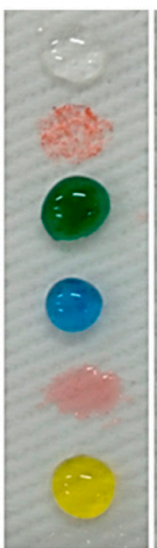

B, 600

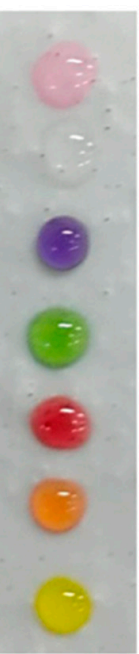

B, 600

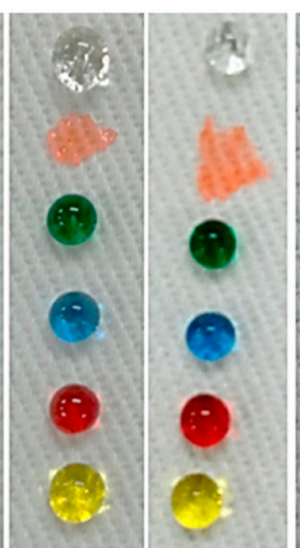

C, 600

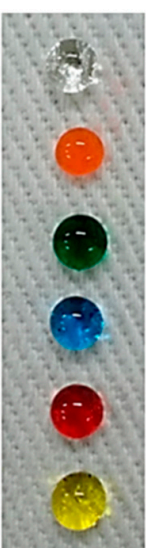

D, 600

$E, 600$
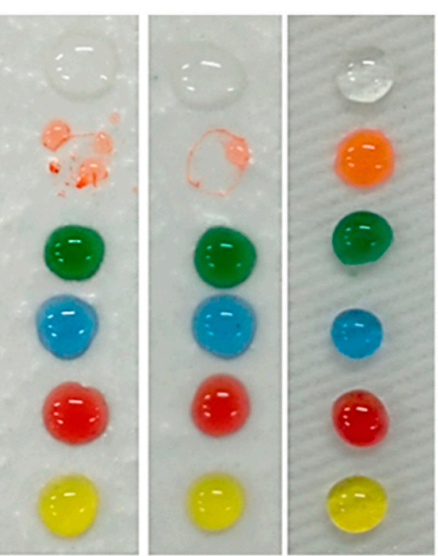

Castor oil

n-Heptane

Paraffin oil

n-Hexadecane

n-Decane

Vegetable oil

$F, 600 \mathrm{G}, 600 \mathrm{H}, 600$
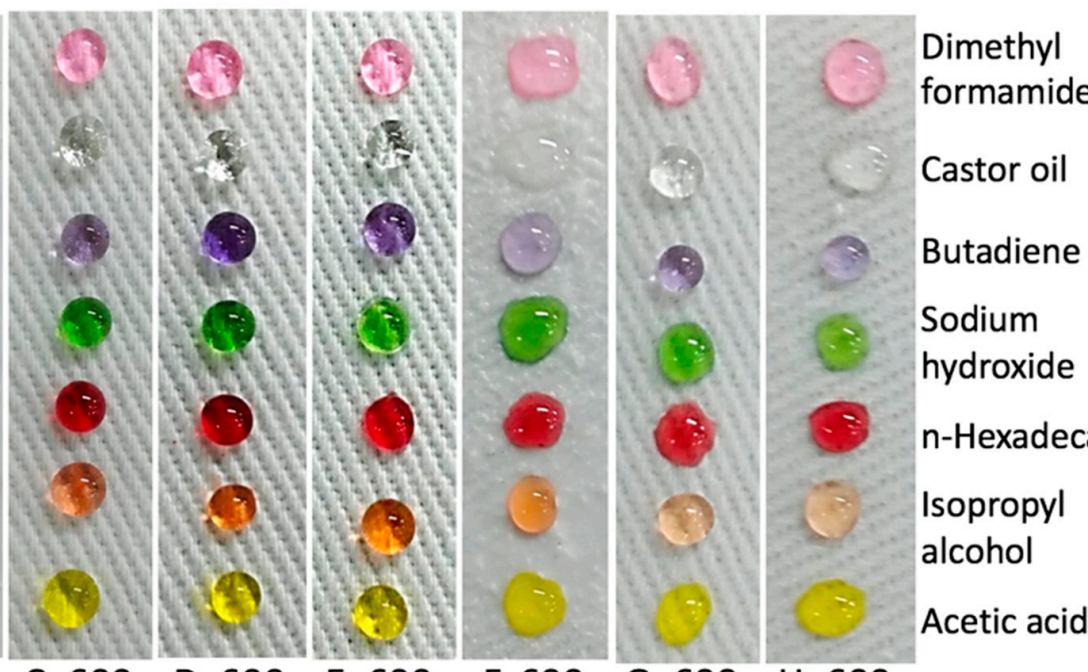

Castor oil

Butadiene

Sodium hydroxide

n-Hexadecane

Isopropyl alcohol

Acetic acid
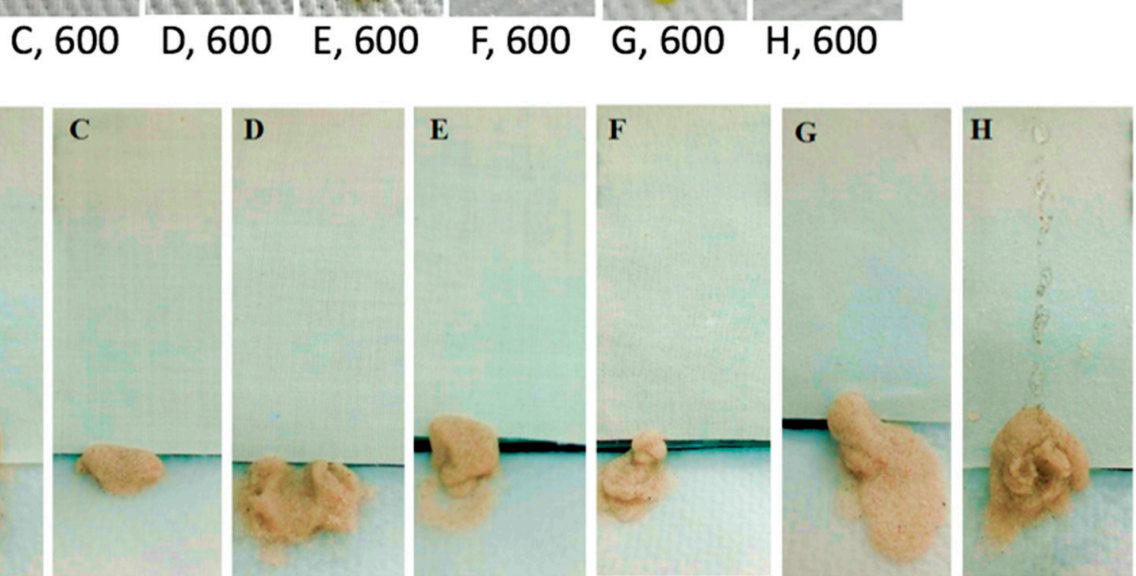

Figure 4. Protection of the as-coated cotton fabrics against oil (a), chemicals (b) and soil (c). 
Table 1. Chemical resistance (s) of uncoated and coated cotton fabrics.

\begin{tabular}{lcccccccc}
\hline \multirow{2}{*}{ Chemicals } & \multicolumn{7}{c}{ Samples } \\
\cline { 2 - 8 } & A & B & C & D & E & F & G & H \\
\hline Acetic acids & 0 & 600 & 600 & 600 & 600 & 600 & 600 & 600 \\
Paraffin oil & 0 & 600 & 600 & 600 & 600 & 600 & 600 & 600 \\
Sodium hydroxide & 0 & 600 & 600 & 600 & 600 & 600 & 600 & 600 \\
n-hexadecane & 10 & 600 & 600 & 600 & 600 & 600 & 600 & 600 \\
Isopropyl alcohol & 0 & 600 & 600 & 600 & 600 & 600 & 600 & 600 \\
Castor oil & 0 & 600 & 600 & 600 & 600 & 600 & 600 & 600 \\
1,4 Butadiene & 0 & 600 & 600 & 600 & 600 & 600 & 600 & 600 \\
Dimethylformamide & 0 & 600 & 600 & 600 & 600 & 600 & 300 & 600 \\
Acetonitrile & 0 & 600 & 600 & 600 & 600 & 600 & 600 & 600 \\
n-decane & 0 & 0 & 600 & 600 & 600 & 600 & 600 & 600 \\
Methanol & 0 & 10 & 600 & 600 & 600 & 186 & 570 & 600 \\
Sulphuric acids & 0 & 0 & 103 & 105 & 107 & 600 & 600 & 540 \\
$n$-heptane & 0 & 0 & 39 & 540 & 600 & 120 & 147 & 600 \\
Triethylamine & 0 & 10 & 535 & 26 & 10 & 540 & 23 & 45 \\
Toluene & 0 & 0 & 600 & 0 & 0 & 600 & 0 & 0 \\
Dichloromethane & 0 & 0 & 0 & 0 & 0 & 60 & 10 & 15 \\
$n$-hexane & 0 & 0 & 0 & 0 & 0 & 0 & 19 & 20 \\
Tetrahydrofuran & 0 & 0 & 0 & 0 & 0 & 18 & 0 & 0 \\
\hline
\end{tabular}

\subsubsection{Soil Resistance}

Soil resistance has been measured to analyze the self-cleaning property of the coated fabrics. Uncoated cotton fabrics are not resistant to soil solution as soil remains on the surface of fabrics as shown in Figure 4c. The soil particles stain on the surface, making it hard to remove them from the surface of the fabrics. All the coated fabrics show excellent soil resistance with an excellent grade 5, and all the soil particles have been absorbed on the tissue papers as seen in Figure 4c. The rubber membrane coated (sample B) and its combination with TMMS and phobol (sample H) are less resistant to soil with the grade 1 . Together with the omniphobicity, the self-cleaning property bring the lotus effect to the coated fabrics $[5,7,33]$, which will see a great application potential in protective clothing such as military uniform, mining uniform and outdoor sportswear.

\subsection{Comfort}

Comfort is another determining factor in developing performance textiles/protective clothing. The coated cotton fabrics show totally different comfort to the uncoated cotton fabrics as shown the results in Figure 5. The thermal resistance (Figure 5a) and water vapor resistance (Figure 5b) of fluoropolymers coated cotton fabrics is not evidently different compared to that of the uncoated cotton fabrics. It is thus evident that the blocking of pores by the formed particles slightly affects the thermal conduction and moisture transfer [42]. However, once rubber membrane is coated onto the surface of cotton fabrics, the thermal and water vapor resistance are so different.

As seen from Figure 5a, the rubber-coated and the combined-coated samples show lower thermal resistances. Besides, the water vapor resistances of these samples are much higher than the rest (Figure $5 b$ ). In this case, the thermal conduction is mainly determined by the materials and the structure of the fabrics. The coating of the rubber membrane has an evident blocking effect on the surface of cotton fabrics, so that the conduction of heat and transportation of moisture (permeability index) through the pores have been blocked. The water vapor resistances of these fabrics are thus higher. However, the thermal conduction is also determined by the materials of the fabrics, and the coated rubber membrane has a higher thermal conduction capability to overweight the blocking of thermal conduction through the fibrous structure [42,43]. The overall thermal resistances of these fabrics are thus lower than the uncoated and fluoropolymers coated ones. 
The moisture management property of cotton fabrics has been changed after the coating. The uncoated cotton fabrics show similar MMT profiles from the top and bottom surfaces, as shown in Figure 5c. Cotton fabrics have excellent wettability, and thus a droplet of water can easily penetrate and spread on both sides of the fabrics. However, the coated fabrics show totally different profiles with a droplet of water on the top surface and nothing on the bottom surface. This is due to the excellent superhydrophobicity of the coating that prevented the water from penetrating and spreading into the fabrics.
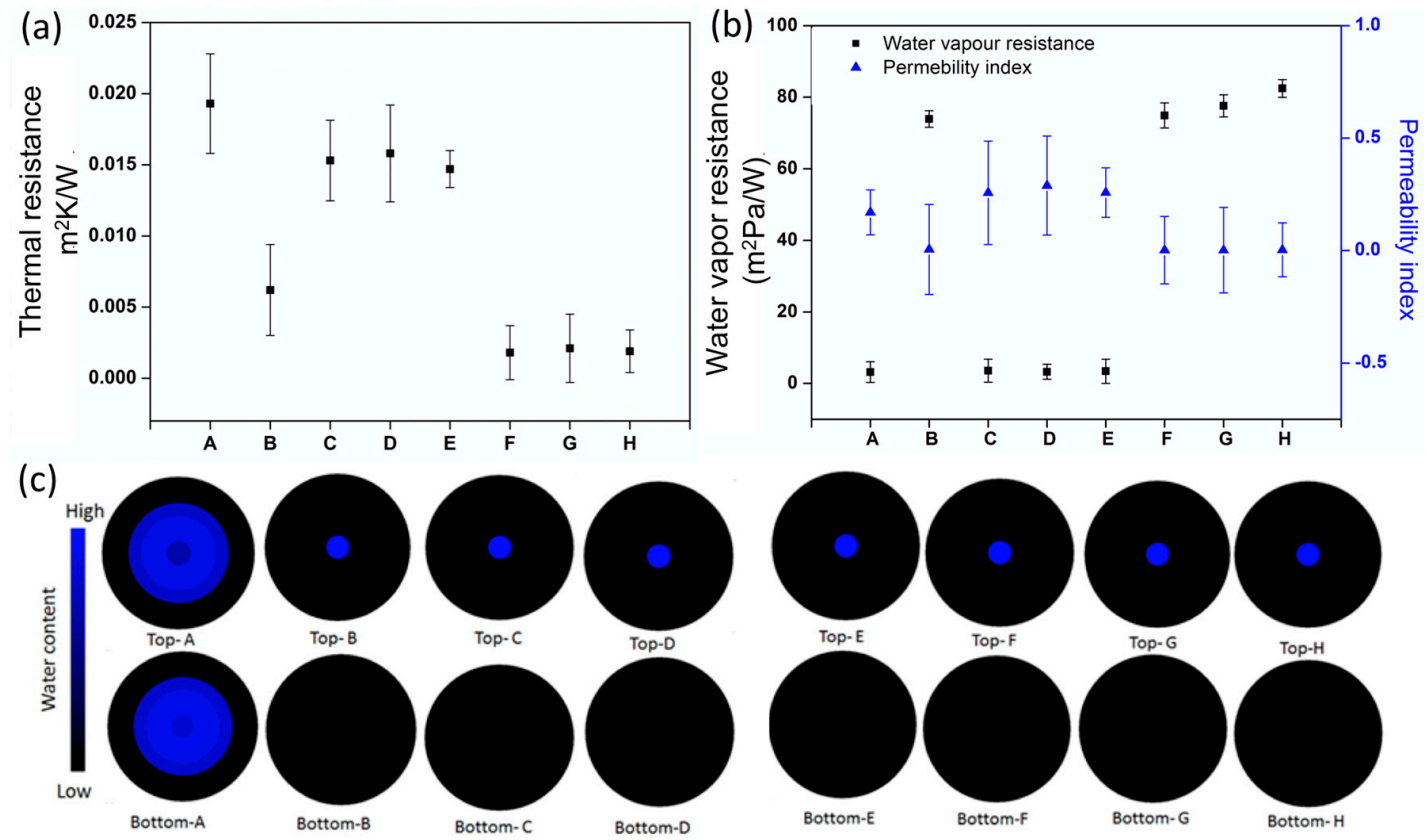

Figure 5. Comfort analysis of the as-coated cotton fabrics: (a) Thermal resistance; (b) water vapor resistance with permeability index; (c) moisture management test (MMT) profiles.

\section{Conclusions}

In the summary, a durable and superomniphobic surface was developed by the pad-knife-padcure coating of rubber membrane and fluoropolymers. The coating brought a thin membrane with a low surface tension and many particles to increase of surface roughness. The coated cotton fabrics were more rigid with a higher bending length, and were stiffer with higher KES values of surface friction and roughness. The coating resulted in a cross-linked network due to the strong bonding between the $\mathrm{CH}_{3}$ groups of rubber and the $\mathrm{CF}_{2}$ groups of fluoropolymers. The coated cotton fabrics showed a water contact angle of around $140-150^{\circ}$, and the superhydrophobicity was durable against different cycles of laundering and pilling. The coated fabrics repelled $80-90 \%$ of water with air permeability of around $70 \%$, and all the aqueous liquids with different water/alcohol ratios were repelled from the coated surface. The coated cotton fabrics showed excellent oil repellency and chemical resistance as most oils and chemicals were repelled from the surface. However, oil and chemicals with closer surface tension to the coated surface were not resistant. The coated fabrics also showed soil resistance without staining of sands on the surface. Due to the existence of the coated layers, the coated fabrics showed decreased thermal resistance and increased water vapor resistance with quite different MMT profiles compared to the uncoated fabrics.

Acknowledgments: Technical assistances from Arun Vijayan are greatly appreciated.

Author Contributions: X.W. and R.P. conceived the ideas and designed the experiments; A.M. performed the experiments; X.W. and A.M. analyzed the data and wrote the paper.

Conflicts of Interest: The authors declare no conflict of interest. 


\section{References}

1. Li, S.; Huang, J.; Chen, Z.; Chen, G.; Lai, Y. A review on special wettability textiles: Theoretical models, fabrication technologies and multifunctional applications. J. Mater. Chem. 2017, 5, 31-55. [CrossRef]

2. Owen, M.J.; Dvornic, R. Surface Applications of Silicones, in Silicone Surface Science; Springer: Berlin, Germany, 2012; pp. 355-374.

3. Xiang, D.; Liu, L.; Liang, Y. Effect of hard segment content on structure, dielectric and mechanical properties of hydroxyl-terminated butadiene-acrylonitrile copolymer-based polyurethane elastomers. Polymer 2017, 132, 180-187. [CrossRef]

4. Holme, I. Innovative technologies for high performance textiles. Color. Technol. 2007, 123, 59-73. [CrossRef]

5. Chen, L.; Wu, F.; Li, Y.; Wang, Y.; Si, L.; Lee, K.I.; Fei, B. Robust and elastic superhydrophobic breathable fibrous membrane with in situ grown hierarchical structures. J. Membr. Sci. 2018, 547, 93-98. [CrossRef]

6. Wang, H.; Xue, Y.; Ding, J.; Feng, L.; Wang, X.; Lin, T. Durable, Self-Healing Superhydrophobic and Superoleophobic Surfaces from Fluorinated-Decyl Polyhedral Oligomeric Silsesquioxane and Hydrolyzed Fluorinated Alkyl Silane. Angew. Chem. Int. Ed. 2011, 50, 11433-11436. [CrossRef] [PubMed]

7. Zeng, C.; Wang, H.; Zhou, H.; Lin, T. Self-cleaning, superhydrophobic cotton fabrics with excellent washing durability, solvent resistance and chemical stability prepared from an SU-8 derived surface coating. RSC Adv. 2015, 5, 61044-61050. [CrossRef]

8. Emelyanenko, A.M.; Boinovich, L.B.; Bezdomnikov, A.A.; Chulkova, E.V.; Emelyanenko, K.A. Reinforced Superhydrophobic Coating on Silicone Rubber for Longstanding Anti-Icing Performance in Severe Conditions. ACS Appl. Mater. Interfaces 2017, 9, 24210-24219. [CrossRef] [PubMed]

9. Shin, Y.; Yoo, D.I.; Son, K. Development of thermoregulating textile materials with microencapsulated phase change materials (PCM). IV. Performance properties and hand of fabrics treated with PCM microcapsules. J. Appl. Polym. Sci. 2005, 97, 910-915. [CrossRef]

10. Lu, X.; Sun, Y.; Chen, Z.; Gao, Y. A multi-functional textile that combines self-cleaning, water-proofing and VO2-based temperature-responsive thermoregulating. Sol. Energy Mater. Sol. Cells 2017, 159, $102-111$. [CrossRef]

11. Pan, S.; Kota, A.K.; Mabry, J.M.; Tuteja, A. Superomniphobic surfaces for effective chemical shielding. J. Am. Chem. Soc. 2012, 135, 578-581. [CrossRef] [PubMed]

12. Horrocks, A.R. High Performance textiles for Heat and Fire Protection A2-Lawrence, Carl A. In High Performance Textiles and Their Applications; Elsevier: Cambridge, UK, 2014; pp. 144-175.

13. Park, Y.; Kim, Y.; Baluch, A.H.; Kim, C.G. Empirical study of the high velocity impact energy absorption characteristics of shear thickening fluid (STF) impregnated Kevlar fabric. Int. J. Impact Eng. 2014, 72, 67-74. [CrossRef]

14. Thakare, V.B.; Tripathi, N.K.; Singh, V.V.; Sathe, M.; Singh, B. Activated Carbon Fabric: An Adsorbent Material for Chemical Protective Clothing. Def. Sci. J. 2017, 68, 83-90. [CrossRef]

15. Fang, J.; Wang, H.; Wang, X.; Lin, T. Superhydrophobic nanofibre membranes: Effects of particulate coating on hydrophobicity and surface properties. J. Text. Inst. 2012, 103, 937-944. [CrossRef]

16. Tan, S.; Li, J.; Gao, G.; Li, H.; Zhang, Z. Synthesis of fluoropolymer containing tunable unsaturation by a controlled dehydrochlorination of $\mathrm{P}$ (VDF-Co-CTFE) and its curing for high performance rubber applications. J. Mater. Chem. 2012, 22, 18496-18504. [CrossRef]

17. DeCoste, J.B.; Peterson, G.W. Preparation of hydrophobic metal-organic frameworks via plasma enhanced chemical vapor deposition of perfluoroalkanes for the removal of ammonia. J. Vis. Exp. 2013, 80, 1-8. [CrossRef] [PubMed]

18. Satam, D.; Lee, H.J.; Wilusz, E. An approach to mass customization of military uniforms using superoleophobic nonwoven fabrics. AATCC Rev. 2010, 10, 59-63.

19. Singh, A.K.; Singh, J.K. Fabrication of durable superhydrophobic coatings on cotton fabrics with photocatalytic activity by fluorine-free chemical modification for dual-functional water purification. New J. Chem. 2017, 41, 4618-4628. [CrossRef]

20. Moiz, A.; Vijayan, A.; Padhye, R.; Wang, X. Chemical and water protective surface on cotton fabric by pad-knife-pad coating of WPU-PDMS-TMS. Cellulose 2016, 23, 3377-3388. [CrossRef] 
21. Lin, J.; Zheng, C.; Ye, W.; Wang, H.; Feng, D.; Li, Q.; Huan, B. A facile dip-coating approach to prepare $\mathrm{SiO}_{2}$ /fluoropolymer coating for superhydrophobic and superoleophobic fabrics with self-cleaning property. J. Appl. Polym. Sci. 2015, 132, 41458. [CrossRef]

22. Kang, Y.K.; Park, C.H.; Kim, J.; Kang, T.J. Application of electrospun polyurethane web to breathable water-proof fabrics. Fibers Polym. 2007, 8, 564-570. [CrossRef]

23. Schutzius, T.M.; Bayer, I.S.; Tiwari, M.K.; Megaridis, C.M. Novel Fluoropolymer Blends for the Fabrication of Sprayable Multifunctional Superhydrophobic Nanostructured Composites. Ind. Eng. Chem. Res. 2011, 50, 11117-11123. [CrossRef]

24. Nouri, N.M.; Saadat-Bakhsh, M. Fabrication method of large-scale and mechanically durable superhydrophobic silicon rubber/aerogel coating on fibrous substrates. J. Coat. Technol. Res. 2017, 14, 477-488. [CrossRef]

25. Seitz, V.; Arzt, K.; Mahnel, S.; Rapp, C.; Schwaminger, S.; Hoffstetter, M.; Wintermantel, E. Improvement of adhesion strength of self-adhesive silicone rubber on thermoplastic substrates-Comparison of an atmospheric pressure plasma jet (APPJ) and a Pyrosil ${ }^{\circledR}$ flame. Int. J. Adhes. Adhes. 2016, 66, 65-72. [CrossRef]

26. Kang, E.T.; Tan, K.L.; Kato, K.; Uyama, Y.; Ikada, Y. Surface Modification and Functionalization of Polytetrafluoroethylene Films. Macromolecules 1996, 29, 6872-6879. [CrossRef]

27. Hekster, F.M.; De Voogt, P.; Pijnenburg, A.M.C.M.; Laane, R.W.P.M. Perfluoroalkylated Substances: Aquatic Environmental Assessment; University of Amsterdam: Amsterdam, The Netherlands, 2002.

28. Haile, M.; Fincher, C.; Fomete, S.; Grunlan, J.C. Water-soluble polyelectrolyte complexes that extinguish fire on cotton fabric when deposited as pH-cured nanocoating. Polym. Degrad. Stab. 2015, 114, 60-64. [CrossRef]

29. Carsio, F.; Alongi, J. Few durable layers suppress cotton comustion due to the joint combination of layer by layer assembly and UV-curing. RSC Adv. 2015, 5, 71482-71490. [CrossRef]

30. Alongi, F.; Carsio, F. All-inorganic intumescent nanocoating containing montmorillonite nanoplatelets in ammonium polyphosphate matrix capable of preventing cotton ignition. Polymers 2016, 8, 430. [CrossRef]

31. Haile, M.; Leistner, M.; Sarwar, O.; Toler, C.M.; Henderson, R.; Grunlan, J.C. A wash-durable polyelectrolyte complex that extinguishes flames on polyester-cotton fabric. RSC Adv. 2016, 6, 33998-34004. [CrossRef]

32. Moiz, A.; Padhye, R.; Wang, X. Coating of TPU-PDMS-TMS on Polycotton Fabrics for Versatile Protection. Polymers 2017, 9, 660. [CrossRef]

33. Dhiman, G.; Chakraborty, J.N. Soil release performance of cotton finished with oleophobol CPR and CMC-Na salt. Fash. Text. 2014, 1, 23. [CrossRef]

34. Yüksekkaya, M.E.; Howard, T.; Adanu, S. Influence of the fabric properties on fabric stiffness for the industrial fabrics. J. Text. Appar. Tekst. Konfeksiyon 2008, 18, 263-267.

35. Ye, W.; Xin, J.H.; Li, P.; Lee, K.L.D.; Kwong, T.L. Durable antibacterial finish on cotton fabric by using chitosan-based polymeric core-shell particles. J. Appl. Polym. Sci. 2006, 102, 1787-1793. [CrossRef]

36. Baek, D.K.; Khonsari, M.M. Fretting behavior of a rubber coating: Effect of temperature and surface roughness variations. Wear 2008, 265, 620-625. [CrossRef]

37. Jassal, M.; Khungar, A.; Bajaj, P.; Sinha, T.J.M. Waterproof breathable polymeric coatings based on polyurethanes. J. Ind. Text. 2004, 33, 269-280. [CrossRef]

38. Vazquez, F. Silicone Softeners for Stain Repellent and Stain Release Fabric Finishing; Dow Corning Corporation: Midland, MI, USA, 2005.

39. Jarvis, N.; Zisman, W. Surface Chemistry of Fluorochemicals; U.S. Naval Research Laboratory: Washington, DC, USA, 1965; pp. 1-34.

40. Lee, L.-H. Wettability and conformation of reactive polysiloxanes. J. Colloid Interface Sci. 1968, 27, 751-760. [CrossRef]

41. Liang, L.; Ruckenstein, E. Pervaporation of ethanol-water mixtures through polydimethylsiloxane-polystyrene interpenetrating polymer network supported membranes. J. Membr. Sci. 1996, 114, 227-234. [CrossRef] 
42. Li, N.; Zeng, F.; Wang, Y.; Qu, D.; Hu, W.; Luan, Y.; Dong, S.; Zhang, J.; Bai, Y. Fluorinated polyurethane based on liquid fluorine elastomer (LFH) synthesis via two-step method: The critical value of thermal resistance and mechanical properties. RSC Adv. 2017, 7, 30970-30978. [CrossRef]

43. Rasid, H.M.; Azhar, N.H.A.; Yusoff, S.F.M. Physicochemical properties of liquid natural rubber bearing fluoro groups for hydrophobic surfaces. J. Polym. Res. 2017, 24, 106. [CrossRef] 